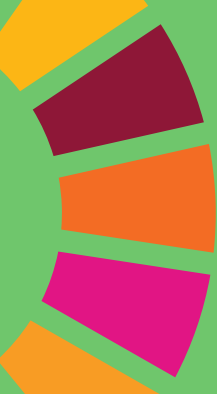

\title{
LAS ALIANZAS, MOTOR IMPRESCINDIBLE PARA ALCANZAR LOS RETOS DE LA AGENDA 2030
}

\section{PARTNERSHIPS, ESSENTIAL ENGINE TO ACHIEVE THE CHALLENGES OF THE 2030 AGENDA}

\author{
Agustín Delgado Martín \\ Iberdrola \\ adelgadom@iberdrola.es \\ Mónica Oviedo Céspedes \\ Iberdrola \\ moviedo@iberdrola.es
}

\section{RESUMEN}

Iberdrola, líder mundial en energías renovables, ha integrado la Agenda 2030 en su estrategia y en su sistema de gobierno corporativo, convirtiéndola en el eje central en la toma de decisiones. Los 17 objetivos que la conforman suponen un plan estratégico para el sector privado, representando la única oportunidad de crecimiento en el medio y largo plazo.

El sector privado debe trabajar en alianza: el trabajo en alianza multiactor recogido en el ODS 17 es imprescindible para poder alcanzar el resto de objetivos. Son indudables los beneficios de los partenariados y resultan obvias la credibilidad y licencias para operar de una actividad que haya sido consensuada y desarrollada entre múltiples y diferentes actores.

Palabras clave: Agenda 2030, Alianzas, Objetivos de desarrollo sostenible, Innovación, Colaboración.

\section{ABSTRACT}

Iberdrola, world leader in renewable energy has integrated the 2030 Agenda into its strategy and corporate governance system, making it the central axis in decision making. These seventeen objectives represent a strategic plan for the private sector that means the only opportunity for growth in the medium and long term. 
The private sector must work in partnership: work in a multi-stakeholder alliance included in SDG 17 is essential to achieve the rest of the objectives. The benefits of the partnerships are unquestionable, the credibility and licenses to operate of an activity that has been agreed and developed between multiple and different actors are obvious.

Keywords: 2030 Agenda, Partnerships, Sustainable development goals, Innovation, Collaboration.

Agustín Delgado. Ingeniero industrial con veinte años de experiencia, comenzó su carrera en diferentes empresas de los sectores ferroviario y energético. Se unió a Iberdrola en 2006 como Director de Innovación. En 2010 fue nombrado Director de Innovación y Sostenibilidad del Grupo Iberdrola. También es responsable del programa de capital de riesgo en IBERDROLA (PERSEO), donde coordina la inversión de PERSEO en empresas tecnológicas relacionadas con nuevas energías sostenibles y modelos de negocio.

Mónica Oviedo. Mónica Oviedo lleva más de 20 años trabajando en Iberdrola y en la actualidad es la responsable de Gestión de Sostenibilidad del grupo. Con la aprobación de los Objetivos de Desarrollo Sostenible por Naciones Unidas en 2015 trabaja desde el área de sostenibilidad para promover la importancia del sector privado, y en concreto de Iberdrola, en la consecución de dicho plan estratégico universal. Coordina el Programa Electricidad Para Todos, lanzado en 2014 para promover iniciativas de acceso universal a la energía eléctrica.

\section{INTRODUCCIÓN GENERAL: EL ROL DE LAS EMPRESAS Y LOS ODS}

Los Objetivos de Desarrollo Sostenible (en adelante, ODS) son el marco común para poder abordar de manera conjunta los desafíos más importantes a los que se enfrenta la humanidad en el siglo XXI. Una de las características más positivas de la Agenda 2030 es que ha sido elaborada de manera participativa entre todos los sectores de la sociedad, siendo la premisa común "no dejar a nadie atrás".

Es por ello que, a diferencia del proceso de diseño y desarrollo de los Objetivos del Milenio (ODM, 20002015), el sector empresarial ha estado involucrado durante todo el periodo denominado post-2015 que arrancó tras la Conferencia de Río+20 de las Naciones Unidas sobre Desarrollo Sostenible, dando lugar a la Agenda 2030, formada por 17 ODS y 169 metas.

El sector empresarial también tuvo una presencia relevante en la Conferencia de Addis Abeba sobre la Financiación al Desarrollo, así como en las reuniones preparatorias. Los temas principales que se trataron en relación al sector privado fueron: la financiación de las infraestructuras, las pequeñas y medianas empresas e inversión responsable y las consideraciones ambientales, sociales y de buen gobierno a lo largo de la cadena de inversión (Naciones Unidas, 2015).

La necesidad de trabajar en alianza para lograr los ODS (objetivo $n^{\circ} 17$ ) se ha definido como parámetro clave y transversal para conseguir el éxito en la integración de la Agenda en todos los sectores.

Se propone así utilizar las tres dimensiones (llustración 1) de sostenibilidad relacionadas a los ODS, siendo éstas: Sostenibilidad ambiental, Inclusión social y Desarrollo económico. 


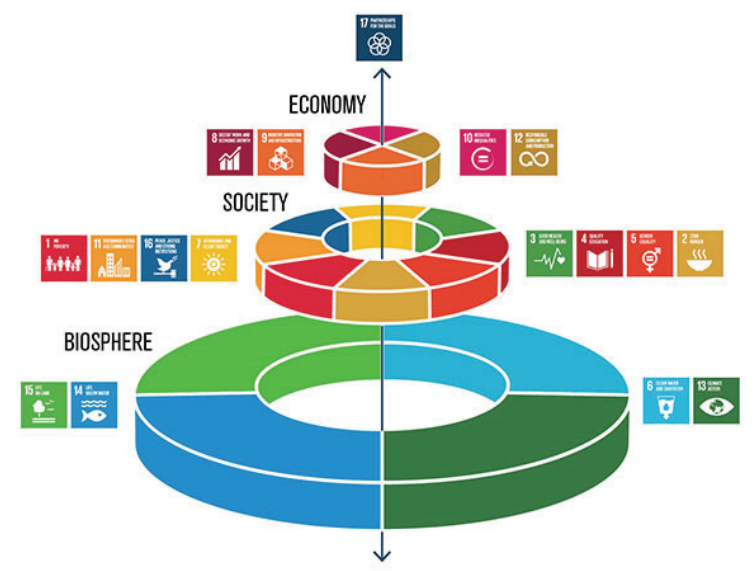

\section{Ilustración 1}

Fuente: https://www.stockholmresilience.org/research/research-news/2016-06-14-how-food-connects-all-the-sdgs.html

Con la aprobación de los ODS las empresas tienen la oportunidad de alinearse y anticiparse a cambios que se están produciendo y que tienen que llegar para conseguir los retos planteados.

El alineamiento puede tener varias formas: con grandes flujos financieros, con futuras políticas públicas y con necesidades sociales emergentes, sobre todo, de quienes van a ser los consumidores del futuro, que masivamente están en los países más poblados, emergentes, donde la tasa de crecimiento es muy fuerte.

Además, los ODS en el sector empresarial suponen una oportunidad para innovar y generar nuevos negocios. Por un lado, poner los ODS en el centro de la organización puede permitir encontrar nuevas trayectorias de innovación. Hay oportunidades inmensas en esta Agenda para que las empresas pongan el foco hacia lo que realmente nos puede permitir tener una vida mejor. Por otro lado, los avances en soluciones sostenibles pueden permitir generar negocios nuevos tanto en los contextos más desarrollados como en los países de rentas más bajas, siendo en estos últimos más probable que la transformación hacia la sostenibilidad pueda darse de manera más rápida, saltándose los errores cometidos por las economías desarrolladas.

No se debe olvidar que, al integrar los ODS en sus estrategias, las empresas tienen la oportunidad de reforzar el compromiso interno de sus trabajadores, retener talento y responder a las expectativas de los grupos de interés. Harvard Business Review (2013) realizó un estudio sobre los motivadores de los trabajadores que se incorporan a las empresas con mejores resultados en bolsa. Este estudio reveló que los tres principales eran: Mastery, es decir, hacer cosas que supongan un desafío importante desde el punto de vista de despliegue de la creatividad, construcción de algo nuevo, etc.; Membership, es decir, pertenencia a una organización y a un equipo de personas con las que es deseable trabajar; y Meaning, es decir, hacer cosas con propósito y que tengan realmente un sentido para la persona. Esto significa que el incentivo extrínseco del dinero no es suficiente para retener el talento. Por eso, una empresa que trabaja en alianza con otras organizaciones, que se plantea una respuesta real a las necesidades de los grupos de interés, que comparte lo que hace en términos de ODS y que amplía el cerebro colectivo de su organización tiene un potencial de estímulo muy importante y puede lograr que la empresa sintonice con las aspiraciones internas de sus empleados, la sociedad y la época en la que vive. 


\subsection{ORGANIZACIONES PARA EL SECTOR PRIVADO}

Es necesario destacar dos plataformas en las que el sector privado participa activamente con un rol colaborativo de todos los actores involucrados en el reto que supone la Agenda 2030.

El Pacto Mundial de las Naciones Unidas (Global Compact) es una iniciativa internacional que tiene por objetivo movilizar a las empresas para contribuir a un mundo más sostenible. Esta red apoya a las empresas para que hagan negocios de una manera responsable, siguiendo diez principios que han elaborado en materia de derechos humanos, trabajo, medioambiente y lucha contra la corrupción. A través del Pacto Mundial, el sector empresarial ha realizado aportaciones que han contribuido a la elaboración de la Agenda post2015 a través de informes dirigidos al Secretario General de Naciones Unidas (Global Compact, 2013). En el ámbito nacional, en 2014 se creó un grupo de trabajo con la finalidad de identificar las prioridades del sector empresarial español respecto de la Agenda post-2015 y canalizar esfuerzos para contribuir a la misma.

La Red de Soluciones para el Desarrollo Sostenible (SDSN, por sus siglas en inglés) es una iniciativa creada en 2012 e impulsada por Naciones Unidas. Esta red cuenta con expertos de la academia, de la sociedad civil y del sector empresarial encargados de dar soluciones técnicas y científicas para el desarrollo sostenible a través de doce grupos temáticos. Uno de los grupos temáticos hace referencia explícita al sector privado: "Redefiniendo el Papel de las Empresas para el Desarrollo Sostenible".

\section{LAS ALIANZAS COMO PIEZA FUNDAMENTAL PARA EL DESARROLLO DE LA AGENDA 2030}

La implicación empresarial es imprescindible para alcanzar la transformación global hacia un escenario sostenible, y, gracias a los ODS, las empresas han visto reconocido su papel como agentes de desarrollo y es material en cualquier discusión que se plantee sobre el futuro económico, social y medioambiental de la humanidad. Dicho esto, es condición necesaria pero no suficiente, ya que la participación del resto de actores de la sociedad civil (gobiernos, academia, ONG, etc.) es vital, necesaria e imprescindible. Cabe destacar que tanto los roles que juega cada actor, así como sus responsabilidades, son diferenciados, y como enfatiza el Objetivo de Desarrollo Sostenible (ODS) número 17, las metas de la Agenda 2030 sólo podrán alcanzarse combinando recursos.

Trabajar en alianzas implica un gran compromiso y la puesta en común de resultados y recursos, algo que no es tarea fácil. Sin embargo, cuando la inversión de todos en tiempo y energía es la correcta, el valor añadido de estas relaciones entre diferentes actores se traduce en un incremento exponencial en las posibilidades de producir un cambio en los sistemas que los rodean.

Los gobiernos tienen instrumentos a su disposición para el impulso de los ODS, desde la elaboración de regulaciones y leyes hasta el desarrollo de políticas de comunicación pública que contribuyan a generalizar el conocimiento y la adhesión a los ODS.

Por ejemplo, el Gobierno de España presentó ante Naciones Unidas en julio de 2018 su Plan de Acción, y entre las diferentes metas que figuraban en él, la Meta Transformadora número cuatro establecía que en 2020 el 100\% de la población española debería conocer la Agenda 2030 y estar sensibilizada sobre el alcance de las transformaciones que conlleva. 
En este sentido, es destacable el papel dinamizador de las universidades. Además de su misión de crear y transmitir conocimiento, están convocadas a involucrar a la sociedad en la generación y aplicación del mismo, siempre con un enfoque interdisciplinar orientado a resolver el tipo de problemas complejos que requiere el cumplimiento de la Agenda.

Es importante destacar también el rol de cada persona. Todos los individuos debemos replantearnos nuestro estilo de vida, así como las decisiones que tomamos cuando desempeñamos distintos papeles en la sociedad. Como consumidores, trabajadores, votantes y ahorradores tenemos el poder de premiar o castigar los comportamientos, sostenibles o no, de diversos tipos de organizaciones.

Naciones Unidas publicó en 2018 la Guía de 170 acciones diarias para transformar nuestro mundo. En un material muy gráfico se recogen diez consejos útiles por cada uno de los Objetivos de Desarrollo Sostenible.
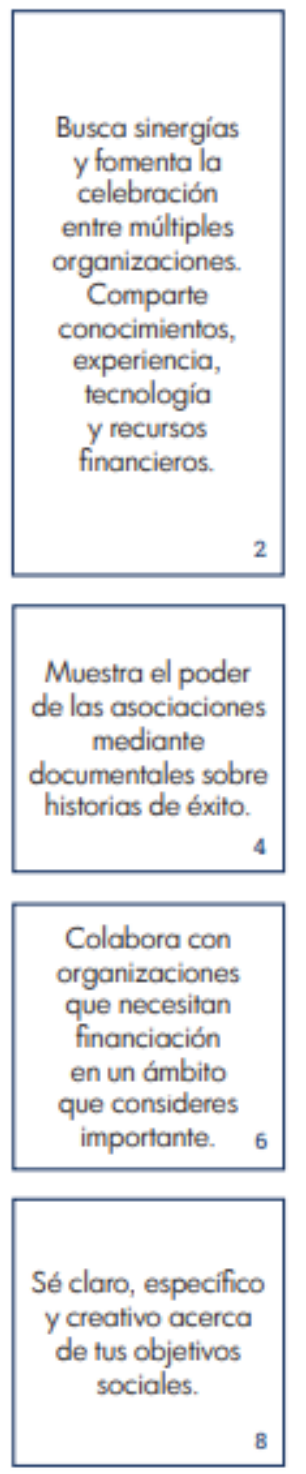
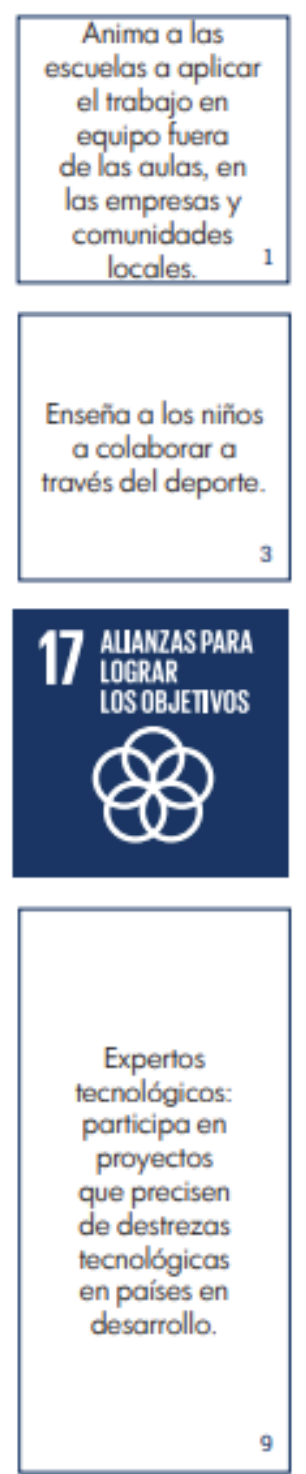
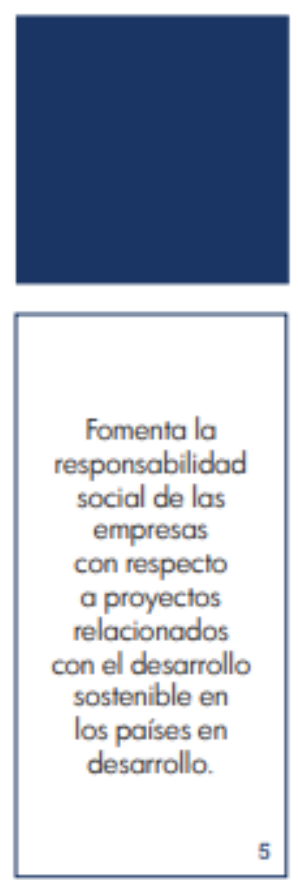

Practica el trabajo en equipo en casa. Comparte actividades entre todos los miembros de la familia y fuera de la familia. 7

Colabora con organizaciones de diferentes paises con las que compartas objetivos.

\section{Ilustración 2}

Acciones referentes al ODS 17 recogidas en la Guía de Naciones Unidas: "170 acciones diarias para transformar el mundo" 
En lo que respecta al sector empresarial, el logro de los ODS depende de su implicación para dar respuestas conjuntas ante los problemas complejos que plantea la Agenda 2030. Especialmente, las grandes empresas tenemos una responsabilidad importante e ineludible, en consonancia con su impacto en la sociedad. En este sentido, pueden destacarse tres razones que explican la necesidad de la participación del sector privado:

En primer lugar, la magnitud de los cambios necesarios: la implicación de las empresas es esencial debido a la necesidad de cambios a gran escala. Las ONG y los gobiernos solos no van a ser capaces de dar una respuesta acorde a la ambición de los problemas que plantea la Agenda. Es ahí donde el sector empresarial, con su capacidad para escalar soluciones a nivel global, puede desarrollar soluciones conjuntas con los diferentes actores para tener el impacto a la escala requerida.

La Agenda implica, en segundo lugar, la obligatoriedad de incluir cambios estructurales en el modelo de producción y de consumo, buscando nuevas formas de vivir más acorde y deseable con las necesidades de nuestro planeta y la sociedad internacional. Este requerimiento va a ser reflejado en las empresas con la introducción de modelos de negocio más sostenibles, ejerciendo como tractores en sus cadenas de valor, proveedores y clientes, y va a ser un determinante esencial para el logro de los ODS relacionados con la producción y consumo responsable.

En tercer lugar, la innovación es esencial para dar respuesta a los retos complejos que plantea la Agenda y que, a día de hoy, no conocemos la manera de solucionarlos. La innovación es abierta y tiene éxito cuando se logra aprovechar el conocimiento periférico de las diferentes organizaciones y ahí, el conocimiento experto del sector empresarial es un valor diferencial.

\subsection{RETOS}

Una vez revisado el papel de muchos de los actores, uno de los desafíos más importantes en el trabajo en alianza multiactor es ponerlo en práctica. Teorizar sobre ello es bastante fácil, lo difícil es llevarlo a la acción. Para comenzar, es necesario acordar su enfoque y garantizar que sus actividades vayan dirigidas a este objetivo, puesto que las metas e intereses de sus distintos participantes varían según el contexto, por lo que se requiere de unas buenas dosis de coraje, paciencia y determinación.

Principales retos:

- No existe regulación enfocada al trabajo en alianza, es decir, hay una falta de normas e instrumentos legales, administrativos y financieros para estimular y promover el trabajo en alianza, lo que dificulta muchísimo y supone trabas administrativas y legales que deben ser superadas muchas veces

- Es habitual que las alianzas se vean limitadas, generalmente esto pasa por motivos como la incapacidad para superar ideas preconcebidas de las partes implicadas, la costumbre de trabajar de forma aislada o un exceso de precipitación durante su creación y desarrollo.

Distintos sectores, trabajando por separado, han desarrollado sus actividades aisladamente, a veces compitiendo entre ellos o duplicando esfuerzos y malgastando recursos valiosos.

- Con frecuencia nos encontramos con un desajuste de inversión en energía y tiempo. Esto ocurre, algunas veces, entre los distintos actores por diferentes motivos: los problemas contextuales de cada uno, como cuestiones específicas de índole regional/nacional político y socioeconómico, gestionar el desequilibrio de poder entre los distintos miembros o la posible falta de transparencia y de rendición de cuentas (en algunos casos). 


\subsection{OPORTUNIDADES}

Son indudables los beneficios del trabajo en alianza, resultan obvias la credibilidad y licencias para operar de una actividad que haya sido consensuada y desarrollada entre múltiples actores.

Así mismo, la alianza proporciona una nueva oportunidad para trabajar mejor en colaboración gracias a su habilidad para reconocer las cualidades y competencias de cada sector y/o actor, así como, encontrar otras formas innovadoras de aprovechamiento de las mismas por el bien común.

Según el informe "Manual de trabajo en Alianza" de la UNDP (United Nations Development Programme) las oportunidades de beneficio común son muy variadas y deben ser destacadas:

- Desarrollo profesional del personal

- Un mejor acceso a la información y a otras redes de trabajo

- Mayor "alcance"

- Mejora de la eficacia operacional

- Productos y servicios más adecuados y efectivos

- Mayor innovación

- Aumento de la credibilidad

- Ampliación del acceso a recursos

\section{CASO IBERDROLA: INTEGRACIÓN ODS}

Con más de 170 años de trayectoria, Iberdrola es hoy un líder energético global, el primer productor eólico y una de las mayores compañías eléctricas por capitalización bursátil del mundo. El grupo suministra energía a cerca de 100 millones de personas en los países en los que está presente.

Lideramos la transición energética hacia un modelo sostenible a través de nuestras inversiones en energías renovables, redes inteligentes, almacenamiento de energía a gran escala y transformación digital para ofrecer los más avanzados productos y servicios a nuestros clientes.

Como resultado de nuestro compromiso medioambiental y nuestra apuesta por la descarbonización de la economía, destacamos como la compañía eléctrica líder en energías renovables reduciendo nuestras emisiones en Europa en un $75 \%$ desde el año 2000 y alcanzando niveles que están cerca de un $75 \%$ menos que la media de las empresas europeas. 


\subsection{ESTRATEGIA ALINEADA CON LA AGENDA 2030}

Estos últimos cuatro años han supuesto un hito en nuestro modelo de gestión y los Objetivos de Desarrollo Sostenible (ODS) han sido una herramienta muy eficaz para el desarrollo de nuestras operaciones y nuestros objetivos en el medio y largo plazo.

Desde el grupo Iberdrola hemos incorporado a nuestra estrategia empresarial y a nuestro Sistema de gobierno corporativo los Objetivos de Desarrollo Sostenible aprobados por la ONU en septiembre de 2015. En consonancia con nuestra actividad, el grupo Iberdrola enfoca su esfuerzo en el suministro de energía asequible y no contaminante (objetivo 7) y la acción por el clima (objetivo 13), y para ello ha creado un plan específico de incentivos a largo plazo.

Además, el grupo contribuye directamente a asegurar agua limpia y saneamiento (objetivo 6), ha aumentado su inversión en actividades de I+D+i (objetivo 9, durante 2018 Iberdrola invirtió más de 260 millones de euros en innovación); promueve el respeto hacia la vida de los ecosistemas terrestres (objetivo 15) y trabaja por establecer alianzas para lograr los objetivos (objetivo 17). La compañía también contribuye de manera indirecta al resto de los Objetivos de Desarrollo Sostenible y para su consecución ha lanzado una primera línea de crédito con indicador sostenible. La aportación del grupo al desarrollo social y económico de las comunidades en las que opera y a la protección del medio ambiente se articula a través de su modelo de negocio energético sostenible.

Para llevar a cabo la correcta integración de los ODS en Iberdrola, ha tenido un papel clave y fundamental la alianza que tenemos con el centro de Innovación y Tecnología para el Desarrollo Humano (itdUPM) de la Universidad Politécnica de Madrid: la Cátedra ODS.

Uno de los principales hitos alcanzados a través de la Cátedra y del trabajo con el itdUPM ha sido llevar a cabo la primera alianza pública privada de Acción Humanitaria de España: La Alianza Shire.

Dicha alianza, compuesta por Iberdrola, Philips Lighting, la Fundación ACCIONA Microenergía, la Agencia Española de Cooperación (AECID) y el itdUPM, desarrolló entre 2014 y 2017 un proyecto piloto en el campo de refugiados de Adi-Harush, Etiopía, con el que se ha mejorado y extendido la red eléctrica que ayuda al funcionamiento de la escuela primaria, dos mercados con 36 pequeños negocios, un centro para mujeres y niñas, un centro de formación y varias cocinas comunitarias. También participaron el Alto Comisionado de las Naciones Unidas para los Refugiados (ACNUR) y de la ONG Norwegian Refugee Council.

Desde 2018 se está desarrollando "Alianza Shire 2", un nuevo proyecto, cofinanciado por la Unión Europea, con el objetivo de seguir mejorando las condiciones de vida en los campos de refugiados -y también en las comunidades de acogida- del norte de Etiopía, mediante su acceso a servicios eléctricos, y la creación de oportunidades de empleo y formación. 
Por otro lado, gracias a esta colaboración con el itdUPM y la Cátedra ODS pudimos aplicar y seguir correctamente la guía SDG Compass, cuyos pasos a seguir se describen a continuación:

\subsection{LA GUIIA SDG COMPASS}

En 2015 se publicó esta guía, con la que se quiso ayudar a las empresas a alinear sus estrategias, así como a medir y gestionar su contribución a los ODS. La guía, realizada por Global Reporting Initiative (GRI), Global Compact y el World Business Council for Sustainable Development (WBCSD), presentaba cinco pasos de cara a ayudar a las empresas a maximizar su contribución a los ODS:

i) Comprensión de los ODS: como primer paso, se ayuda a las empresas a familiarizarse con los ODS.

ii) Definiendo prioridades: aprovechar las oportunidades comerciales más importantes que suponen los ODS y reducir riesgos. Se anima a las empresas a definir sus prioridades basadas en una evaluación de sus aspectos positivos y negativos y el impacto actual y potencial en los ODS en sus cadenas de valor.

iii) Establecer objetivos: la fijación de objetivos es fundamental para el éxito empresarial y ayuda a fomentar prioridades compartidas y un mejor rendimiento en toda la organización. Al alinear los objetivos de la empresa con los ODS, se demuestra el compromiso de los líderes con el desarrollo sostenible.

iv) Integración: integrar la sostenibilidad en el negocio principal y en la gobernanza es clave para lograr los objetivos establecidos. Perseguir metas compartidas o abordar desafíos sistémicos supone que las empresas, cada vez más, se asocian a través de sus cadenas de valor, dentro de su sector o con gobiernos y organizaciones de la sociedad civil.

v) Informes y comunicación: los ODS permiten a las empresas reportar información sobre el desempeño del desarrollo sostenible, utilizando indicadores comunes y un conjunto compartido de prioridades.

\subsubsection{COMPRENSIÓN DE LOS ODS}

Remontándonos a 2014, un año antes de la aprobación de la Agenda 2030, el Consejo de Administración de Iberdrola aprobó la Política de gestión de sostenibilidad, basada en cinco ejes prioritarios. Desde el punto de vista de la sostenibilidad energética, hablamos de competitividad, seguridad de suministro y medio ambiente y desde el punto de vista de la sostenibilidad empresarial, consideramos que las decisiones tienen que responder a las vertientes medioambiental, económica y social. En torno a esos cinco bloques definimos un cuadro de mando con los indicadores a monitorear.

En la Dirección de Innovación y Sostenibilidad en la que trabajamos, ya en 2014 comenzamos a tomar conciencia de la relevancia que la Agenda iba a tener para el sector privado y realizamos un primer ejercicio para ver cómo los ODS estaban incluidos en nuestro cuadro de mando para la gestión de la sostenibilidad. Organizamos, como primera medida, una sesión de formación con nuestros directivos a nivel global, en la que destacados miembros del mundo académico resaltaron la importancia de la Agenda y cómo Iberdrola debía utilizarla para buscar oportunidades innovadoras de crecimiento en su negocio. 


\subsubsection{DEFINIENDO PRIORIDADES}

Toda empresa tiene un impacto muy relevante en muchos ODS, pero desde Iberdrola, y dado que somos un referente mundial en el sector energético, no tuvimos ninguna duda en elegir el Objetivo número 7 como el prioritario, dado que reúne, bajo el título de "Energía asequible y no contaminante", tres ejes de actuación esenciales para nosotros: acceso universal, energías renovables y eficiencia energética. Además, y de manera concluyente, el Objetivo 13, de "Lucha contra el cambio climático" está íntimamente ligado al 7. Estos dos son los ODS que consideramos prioritarios y se sitúan en lo alto de nuestra pirámide.

En un segundo nivel tenemos un impacto directo en otros, como los de innovación, biodiversidad, agua y alianzas. Nos gustaría destacar el papel importante que tienen las alianzas para alcanzar los ODS, aunque somos conscientes de que es uno de los objetivos más complicados, puesto que implica un cambio radical en la manera de trabajar y faltan estructuras adecuadas para colaborar multisectorialmente. En un tercer nivel, y de forma indirecta, contribuimos al cumplimiento del resto de los ODS (Ilustración 3).

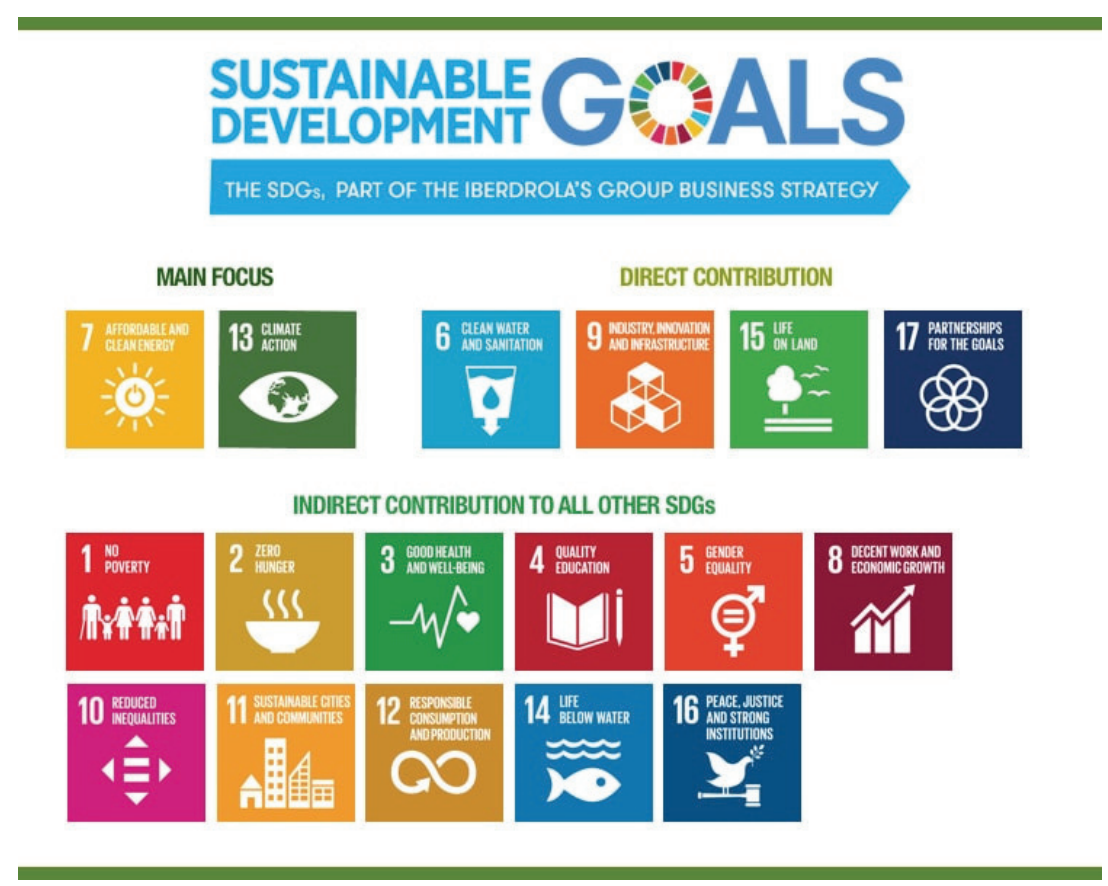

Ilustración 3

Fuente: Elaboración de Iberdrola

\subsubsection{ESTABLECER OBJETIVOS}

Nos gustaría resaltar un elemento innovador diferencial que creemos clave para el éxito de nuestra aportación a la Agenda 2030: el compromiso indiscutible de la alta dirección. Iberdrola ha incluido objetivos concretos vinculados a las metas de los ODS, con referencia temporal de medio y largo plazo, que están ligados a su retribución y repercuten en cascada al resto de los empleados.

Con relación al objetivo de acceso universal incluido en el ODS 7, Iberdrola cuenta con el programa "Electricidad para todos" cuya meta es triplicar el número de beneficiarios del mismo para 2030. 
El programa "Electricidad para todos" es la respuesta de Iberdrola al Ilamamiento que ha hecho la comunidad internacional para extender el acceso universal a formas modernas de energía, con modelos ambientalmente sostenibles, económicamente asumibles y socialmente inclusivos, enlazando su finalidad de manera estratégica con el ODS 7.1.

En el eje de las energías renovables, Iberdrola es un referente mundial en su promoción, con casi 31 gigavatios (GW) de capacidad instalada. Pero, como ya hemos mencionado, la Agenda implica ir un paso más allá y no sería suficiente una aportación vinculada a nuestra actividad ordinaria. Asumiendo dicho liderazgo, en la última revisión del Plan Estratégico hemos planteado un crecimiento muy significativo, de 9 GW adicionales.

Encuanto a la eficiencia energética, estamos firmemente convencidos de que la electrificación de la economía es la vía más rápida y eficiente de alcanzar la descarbonización. Y tenemos datos concretos para ilustrar su aportación: ésta ha evitado la emisión, en los últimos años, de 60 millones de toneladas de $\mathrm{CO}_{2}$. Es necesario seguir avanzando en esa línea, potenciando el autoconsumo solar, la electrificación del calor o la movilidad eléctrica. Así, contamos con objetivos públicos de instalación de puntos de recarga en España y de alcanzar flotas 100\% eléctricas.

Iberdrola fue una de las pocas empresas que acudió a la Cumbre climática de París del año 2015 con objetivos concretos de reducción de la intensidad de emisiones para el año 2030, a pesar de tener ya una intensidad un $40 \%$ menor a la media europea.

Es muy importante resaltar, de nuevo, las consecuencias económicas de la asunción de estos objetivos concretos, dado que la adopción de esta decisión por parte de Iberdrola podría quedar acotada al terreno de los meros deseos y buenas intenciones. En el año 2017 fuimos de las primeras empresas del mundo en incluir dentro de los objetivos de nuestros consejeros algunos directamente vinculados a la contribución al cumplimiento de los ODS.

\subsubsection{INTEGRACIÓN}

El cuarto paso mencionado en la guía SDG Compass sería la integración de la Agenda 2030 en la actividad empresarial. Desde Iberdrola la consideramos clave y nos gustaría detallar cómo la estamos llevando a cabo, a través de multitud de acciones que pasamos a detallar en tres apartados: internalización, medición y reporte y difusión.

i) Internalización en Iberdrola: una de las acciones más relevantes ha sido la reforma de nuestro Sistema de Gobierno Corporativo, aprobada por el Consejo de Administración en 2018 de cara a incorporar al detalle el impacto de la Agenda 2030 en la compañía. La inclusión del Dividendo Social (la respuesta de Iberdrola a los asuntos más relevantes del sector, a las necesidades y expectativas de sus Grupos de interés y a los retos más significativos de las sociedades en las que está presente) y la formulación del Propósito de Iberdrola ("Continuar construyendo, cada día y en colaboración, un modelo energético más eléctrico, saludable y accesible") son dos buenos ejemplos de la importancia de la sostenibilidad para nuestro Consejo.

El grupo Iberdrola ha acuñado el término 'dividendo social' para expresar su objetivo de creación de valor sostenible para sus Grupos de interés mediante el desarrollo de todas sus actividades'.

${ }^{1}$ Más información: https://www.iberdrola.com/conocenos/energetica-del-futuro/grupos-de-interes/creacion-de-valor-dividendo-social 
Otra actividad destacable es la formación a nuestros empleados. Se trata de campañas o iniciativas de sensibilización que provocan un impacto en los empleados, aumentando así su compromiso con la Agenda 2030. Aparte de la ya comentada para directivos, hemos organizado sesiones específicas de ODS (Alianzas en el año 2017, "ODSeina" en el año 2019, etcétera) y tenemos formación virtual en torno a la Agenda para nuestros más de 34.500 empleados. Asimismo, una gran pirámide de los ODS está presente en nuestras sedes, nuestras firmas y tarjetas de identificación incluyen los mismos logos, y, además, todas las noticias que aparecen en el Portal del Empleado llevan vinculado el objetivo al que están apoyando.

En concreto, nos gustaría destacar toda la labor relacionada con el voluntariado corporativo. Desde 2015, las oportunidades de acción social están detalladas por el impacto que conllevan y esta preocupación de medición del impacto ha sido premiada por Naciones Unidas, con el reconocimiento Impact 2030. Una iniciativa que hemos lanzado recientemente, y que animamos a difundir, es la denominada "ODS al cole", mediante la que los empleados de Iberdrola estamos participando en sesiones de formación en los colegios de nuestros familiares y amigos, explicando a los niños la importancia de la Agenda. Creemos que es muy importante que los niños la conozcan para que reclamen a los adultos acciones concretas.

Otra buena práctica reciente ha sido la difusión interna de la guía de Naciones Unidas, que incluye 170 actividades concretas para llevar a cabo en la vida diaria. Pedimos a los empleados que nos manden sus compromisos y les enviamos el famoso pin de los ODS, convirtiendo a los empleados en embajadores de la propia Agenda.

ii) Medición y reporte: Iberdrola lleva a cabo numerosas actividades enfocadas a dar a conocer su aportación a los ODS. Cabe destacar el creciente interés de los inversores ASG (Ambiental, Social y Gobernanza), que están vinculando todo este conjunto de iniciativas con un impacto económico directo, dando visibilidad a la oportunidad de crecimiento que supone para el sector privado su inclusión en los diferentes índices de sostenibilidad: DJSI, FTSE, CDP o Bloomberg. Una iniciativa muy innovadora por parte de lberdrola ha sido el lanzamiento, en marzo de 2019, de la primera línea de crédito, de 1.500 millones de euros, vinculada a metas muy concretas del Objetivo 7.

iii) El último eje correspondiente a la metodología de integración en Iberdrola coincide con el último paso de la guía SDG Compass: difusión. Aprovechamos la capacidad que tiene una gran empresa como la nuestra para poder dar difusión a la Agenda 2030. Además de nuestra participación en múltiples foros, nos gustaría destacar alguna iniciativa concreta, como nuestra encuesta a proveedores: el año pasado preguntamos a nuestros suministradores si conocían los ODS, cuáles eran los que ellos consideraban prioritarios, y les pedíamos trabajar conjuntamente para contribuir a su cumplimiento.

\subsubsection{INFORMES Y COMUNICACIÓN}

Somos un referente por nuestros informes, que resaltan la importancia y vinculación de nuestras actividades con la Agenda, así que animamos a explorar nuestra web corporativa (www.iberdrola.com), donde figura toda la información sobre la actividad de Iberdrola. Además, desde la compañía hemos coorganizado diferentes seminarios y eventos para comunicar avances en el cumplimiento de los ODS: por ejemplo, en 2018 en Salamanca celebramos la Primera Conferencia Iberoamericana sobre la Agenda 2030 y en 2019, en mayo, hemos celebrado, unas jornadas sobre innovación y aceleración (Acelera 2030, www.acelera2030.org) y un hackaton ODS en nuestro Campus de formación de San Agustín del Guadalix. 


\section{CONCLUSIONES}

El sector privado está llamado a jugar un papel fundamental en el reto transformador que supone la Agenda 2030. No debemos olvidar que este nuevo papel tiene que ser desempeñado de forma conjunta con el sector público y el tercer sector para alcanzar los diecisiete retos que tenemos por delante en los próximos diez años.

Iberdrola cree firmemente que el reto de la Agenda supone una transformación radical en la actividad de todos los agentes lo que refrenda el importante papel que damos a la innovación.

La innovación es una variable estratégica para el grupo Iberdrola y constituye la principal herramienta para garantizar la sostenibilidad, la eficiencia y la competitividad de la compañía a la hora de hacer frente a los diferentes retos que se plantean a través de la Agenda 2030 y que, desde Iberdrola, intentamos abordar con un trabajo colaborativo, caracterizado por la co-creación y la innovación abierta.

Iberdrola quiere ser un referente en este nuevo escenario, liderando la innovación necesaria para poder alcanzar este nuevo plan estratégico universal. En concreto, gracias a una estrategia de anticipación y trabajo en alianzas con agentes internos y externos, que transciende a todas las unidades de negocio de la compañía y a todas sus áreas geográficas, Iberdrola es hoy día la utility española más innovadora y la tercera de Europa, según la clasificación de la Comisión Europea. 


\section{REFERENCIAS BIBLIOGRÁFICAS}

- Cuadernos para Ingenieros: Sobre los objetivos de desarrollo sostenible en general y el objetivo 17. Agustín Delgado en colaboración con el ITD (UPM).

- Gobierno de España (2019), "Plan de Acción para la implementación de la Agenda 2030"

- Organización de Naciones Unidas. Guía de 170 acciones diarias para transformar nuestro mundo. Obtenido de: https://www.un.org/sustainabledevelopment/es/wp-content/uploads/sites/3/2018/08/170Actionsweb_Sp.pdf

- Stott, Leda. (2019) “Alianzas para el desarrollo sostenible: cuáles son los retos que debemos superar", en Somos Iberoamérica.

- Tennyson, R. (2003). Manual de trabajo en Alianza. Obtenido de:

https://www.undp.org/content/dam/aplaws/publication/es/publications/capacity-development/thepartnering-toolbook-spanish/Partnering-Toolbook-Spanish. 